\title{
3/4inch HDTV Digital VCR (D6)
}

( TOSHIBA )

\section{Features}

The world first cassette type HDTV digital VTR for professional use High speed : Total data rate of $1.2 \mathrm{Gbps}$ (150Mbps $\times 8$ channels) High density : $6.6 \mu \mathrm{m}^{2} /$ data bit $\rightarrow 4.5 \mathrm{Tbit} /$ cassette

\section{System Specifications}

\begin{tabular}{|l|l|}
\hline Dimensions (WxHxD) & $447 \times 397 \times 700 \mathrm{~mm} / \mathrm{unit}$ \\
\hline Weight & Approx. 55 kg/unit \\
\hline Power requirement & $100-120 \mathrm{~V} / 200-240 \mathrm{~V} \pm 10 \%$ \\
\hline Power consumption & Approx. $1 \mathrm{KW}$ \\
\hline Cassette type & $\mathrm{D} 6(\mathrm{~L}, \mathrm{M}, \mathrm{S})$ \\
\hline Recording time & $\mathrm{L}: 64 \mathrm{~min}, \mathrm{M}: 28 \mathrm{~min}, \mathrm{~S}: 8 \mathrm{~min}$ \\
\hline Video & \\
Sampling frequency & $\mathrm{Y}: 74.25 \mathrm{MHz}$ \\
& $\mathrm{Pb} / \mathrm{Pr}: 37.125 \mathrm{MHz}$ \\
Quantization & $8 \mathrm{bit}$ \\
\hline Audio & \\
Sampling frequency & $48.0 \mathrm{KHz}$ \\
Quantization & $24 \mathrm{bit}(\mathrm{max})$ \\
Channels & 10 \\
\hline Slow-motion & $\pm 0.25(\max )$ \\
\hline Picture search & $\pm 15(\max )$ \\
\hline
\end{tabular}

\section{Perspectives}

Recording density in 2000

$: 2 \mu \mathrm{m}^{2} / \mathrm{bit}$

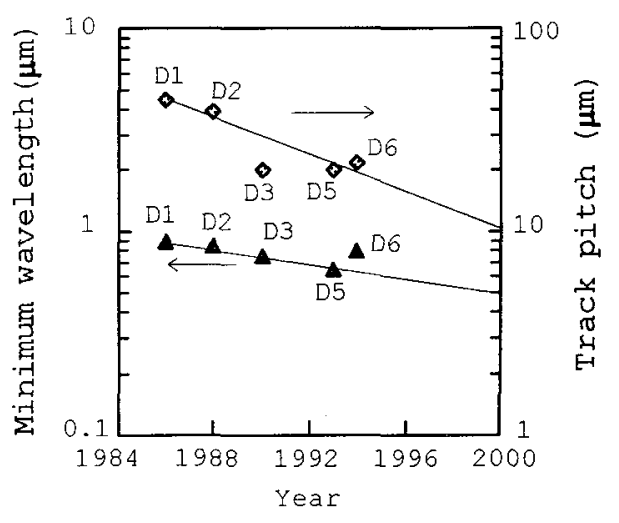




\section{Key Technologies}

\section{Tape format}

- Segment structure (of 8 tracks) and Guard-band between segments

- Applicable to HDTV multi-standard

- Minimum number of erase heads required

- Improvement of error in editing modes

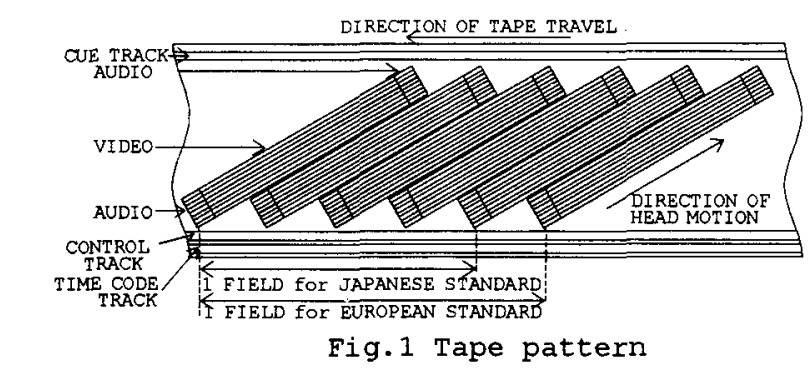

\section{Channel coding}

- 8-12 conversion

- Tmin : 1.33Tb

- Tmax : 5.33Tb

- Minimum wavelength : $0.81 \mu \mathrm{m}$

- Channel rate : $227 \mathrm{Mbps}$

* Tb:original data bit interval

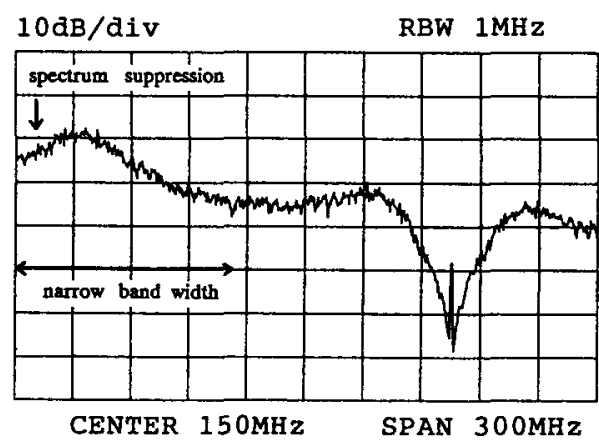

Fig.2 spectrum of 8-12 conversion

\section{Head and Tape}

- High performance laminate head for playback

$\mathrm{S} / \mathrm{N}$ ratio : +3dB compared with ferrite head

- Metal particle tape

Thickness : $11 \mu \mathrm{m}$

$\mathrm{Hc}: 1600 \mathrm{Oe}$

$\mathrm{Br}: 2500$ Gauss

$\mathrm{S} / \mathrm{N}$ ratio : $+2.5 \mathrm{~dB}$ compared with D2 tape

\section{Error correction code}

- Reed-Solomon product code with great capability

- Inner code $:(227,211)$

- Video outer code : $(254,240)$

- Audio outer code : ( 40, 28 ) (doubly recorded for audio)

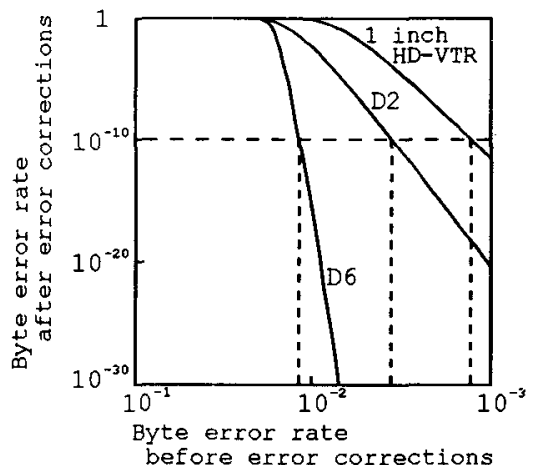

Fig. 3 Comparison of error correction capability for video data

\section{Mechanism}

- 34 heads on rotary drum

- Drum revolution speed : $9000 \mathrm{rpm}$

- Head to tape speed : $46 \mathrm{~m} / \mathrm{s}$

- Average track pitch : $22 \mu \mathrm{m}$

- Track non-linearity : less than $5 \mu \mathrm{m}$

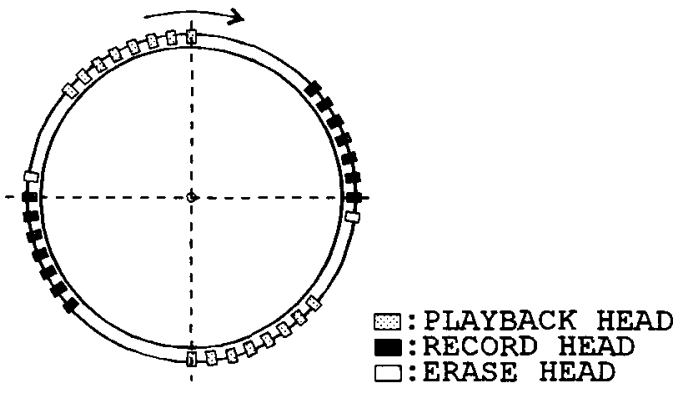

Fig. 4 Head arrangement on rotary drum 